Volumen 25, No 1, Páginas 37-45

IDESIA (Chile) Enero - Abril 2007

\title{
SITUACIÓN SOBRE LAS EXPORTACIONES DE LAS CINCO PRINCIPALES ESPECIES FRUTALES DE CHILE A LOS MERCADOS DE AMÉRICA DEL SUR ENTRE LOS AÑNOS 1993-2004
}

\author{
SITUATION ON THE EXPORTS OF THE FIVE MAIN FRUIT SPECIES \\ FROM CHILE TO THE MARKETS OF SOUTH AMERICA \\ BETWEEN YEARS 1993-2004
}

Luis Tapia Iturrieta ${ }^{1}$

\begin{abstract}
RESUMEN
Desde el año 1993, Chile inicia un activo proceso de firma de Acuerdos de Complementación Económica (ACE) con los diferentes países de América del Sur y el bloque del MERCOSUR, que apuntaban a disponer de socios comerciales para colocar sus exportaciones dentro de un marco preestablecido de preferencias arancelarias. En este estudio se analizan los resultados alcanzados para las cinco principales especies frutales chilenas (uva, manzana, pera, kiwi y palta), las cuales en su mayoría presentan una tendencia a la reducción en la participación de mercado, lo que se manifiesta en una menor presencia de fruta chilena en los mercados de América del Sur, que se expresan en menores proyecciones de ingresos provenientes de esta área; sin embargo, destacan los ACE con Colombia y Ecuador, en donde la fruta chilena mantiene aún una presencia importante.

Palabras clave: Exportaciones de frutas chilenas, Acuerdos de Complementación Económica, comercio de fruta en América del Sur.
\end{abstract}

\section{ABSTRACT}

Since 1993 Chile initiates an active process of signing Economic Integration Treaties with several countries in South America and the MERCOSUR area that aimed to have commercial partners to place its exports within a pre-established frame of tariff preferences. In this research the results reached for the top five Chilean fruit species (grape, apple, pear, kiwi and avocado) are analyzed which in their majority present tendency to the reduction in participation of the market, what is pronounced in a smaller presence of Chilean fruit in the markets of South America, that are showed in smaller projections of incomings in this area. However the agreements with Colombia and Ecuador, where the Chilean Fruit still keep an important presence.

Key words: Chilean fruits exports, Economic Integration Treaties, trade of fruit in South America.

\section{INTRODUCCIÓN}

La política comercial de Chile desde 1990, en relación al sector frutícola, ha apuntado a conseguir acceso a nuevos mercados, donde se establecen algunas preferencias arancelarias. Esta estrategia del país es analizada por CEPAL (2002) donde se registra la serie de Acuerdos de Complementación Económica (ACE) y Tratados de Libre Comercio
(TLC) que Chile ha implementado desde 1993 en adelante, iniciándose con el ACE $\mathrm{N}^{\circ} 23$ con Venezuela, el ACE $\mathrm{N}^{\circ} 22$ con Bolivia, en el año 1994 se establece el ACE N ${ }^{\circ} 24$ con Colombia, en el año 1995 entra en vigencia el ACE $\mathrm{N}^{\circ} 32$ con Ecuador, en año 1996 se establece el ACE No 35 con los países del MERCOSUR, finalmente en el año 1997 se establece el ACE N 38 con Perú.

$1 \quad$ Facultad de Ciencias Agronómicas, Universidad de Tarapacá, 1tapia@uta.cl

Fecha de Recepción: 13 Abril 2006

Fecha de Aceptación: 04 Junio 2006 
Las políticas de ajuste estructural implementadas por muchos países están provocando dificultades en los procesos de integración que experimenta la economía mundial y las acciones de integración vía acuerdos tienden a un nuevo equilibrio entre las economías que se integran (MINIAGRI, 2000).

También la política comercial de Chile se ha caracterizado por una reducción unilateral de aranceles que a la fecha alcanza a un $6 \%$ de arancel de nación más favorecida (NMF) en la Organización Mundial de Comercio (OMC) y en términos reales el nivel arancelario se reduce tan solo de un $1 \%$, al considerar el conjunto del comercio vigente alcanzado en la liberalización, en los marcos de los ACE y TLC.

La importancia relativa de las cinco especies (uva, manzana, palta, kiwi y pera) en las exportaciones frutícolas chilenas está dada, ya que representan el $80 \%$ del valor en los ingresos en las exportaciones (Tapia, 2006), dejando a 12 especies con sólo el $20 \%$ de incidencia durante el período analizado.

Todos estos acuerdos se encuentran en el marco sugerido por la Organización Mundial de Comercio OMC, tendientes a dinamizar el comercio mundial y mejorar las condiciones de acceso a los diferentes mercados. También preocupan las amenazas a las preferencias arancelarias que Chile ha obtenido a la fecha y que en los próximos años se vean diluidas por otros TLC que negocien nuestros socios comerciales con terceros países (Sepúlveda y Rivera, 2004).

El acuerdo con el MERCOSUR despierta alto interés de las exportaciones sectoriales de fruta fresca, hortalizas y frutas procesadas, vinos y productos pecuarios que se manifiestan en un aumento del bienestar social, pero también una serie de transferencias de ingresos entre consumidores y productores, y entre diferentes productores agropecuarios y agroindustriales (Muchnik, et al. 1996).

En el año 2010, según Domínguez (2000), Chile habrá eliminado la gran mayoría de las barreras comerciales, al menos con los países del MERCOSUR y será parte de un gran mercado abierto en el continente; ello trae consigo cambios radicales en la competitividad de ciertos rubros y sectores.

\section{MATERIAL Y MÉTODOS}

Este es un estudio de tipo descriptivo, que tiene por objetivo indagar sobre la incidencia y los valores que se alcanzan en las exportaciones de las principales especies frutales chilenas en los mercados de América del Sur. Además, se especifican las características de la competitividad en cada mercado, evaluando este comportamiento y las relaciones que se producen entre ellos (Hernández, et al. 1998).

Asimismo es una investigación de tipo no experimental, puesto que se realiza sin hacer cambios intencionales en las variables; se observan los cambios y efectos que sucedieron.

Además es un diseño no experimental longitudinal de tendencia, ya que se desea analizar los cambios a través del tiempo, en cuanto a la conducta de los países y las relaciones entre éstos para hacer inferencias respecto al cambio, sus determinantes y consecuencias en distintos momentos en el tiempo.

Más aún, el diseño de la investigación es de tipo documental, con medición de datos a partir de información secundaria obtenida de las bases de datos.

De esta forma se procedió a la recopilación de la información para establecer las series entre 1994-2004, para los ingresos que generan estas exportaciones de las cinco especies a los distintos países de América Latina con los cuales existen ACE; esta información se recopila a partir de la base de datos del Ministerio de Agricultura (ODEPA, 1993-2004).

La información obtenida se tabula en gráficos a fin de determinar la tendencia que presenta en las exportaciones de estas cinco especies frutales chilenas en cada país, para el período analizado, considerado desde cuando entra en vigencia el acuerdo respectivo hasta el año 2004.

\section{RESULTADOS}

En el Gráfico 1 se presenta la secuencia en el tiempo de los diferentes Acuerdos de Complementación Económica que Chile fue firmando con los países de América del Sur desde 1994 en adelante, hasta completar una importante red con el conjunto de países de la región. 


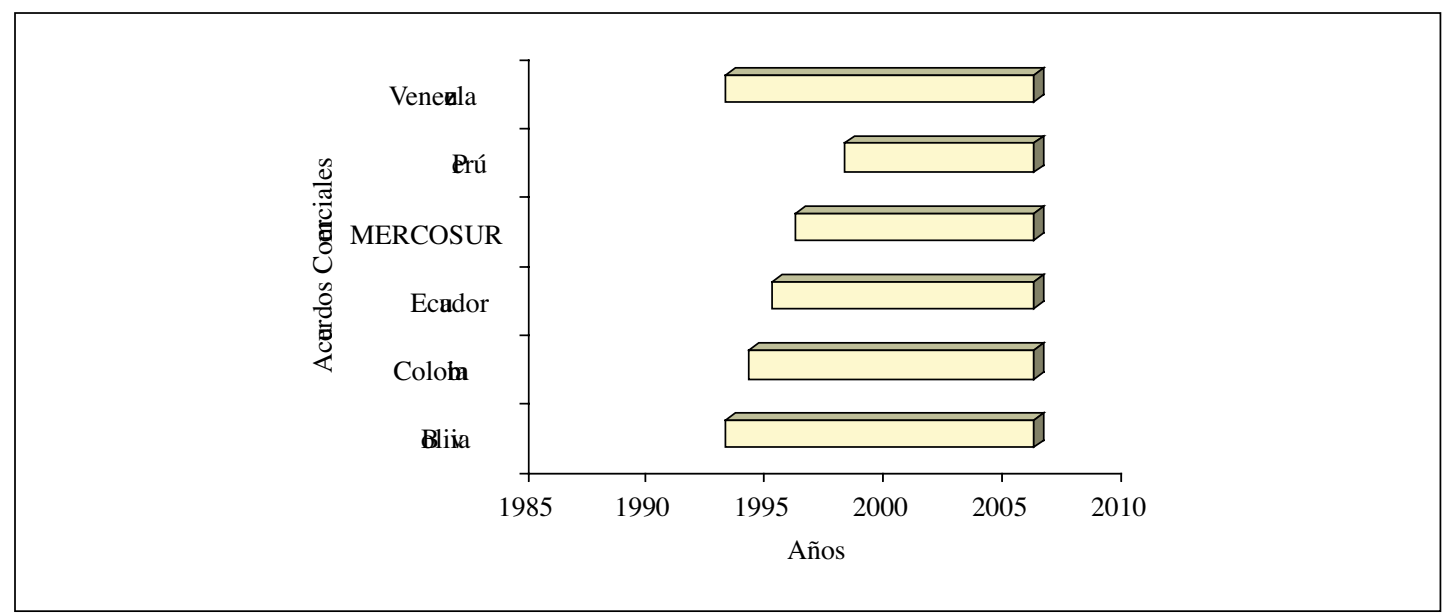

Gráfico 1. Secuencia de los acuerdos comerciales firmados por Chile y los países de América del Sur entre los años 1994-2006.

Fuente: Direcon.

Los acuerdos se encuentran todos vigentes con distintos niveles de desarrollo y diferente potencial para la colocación de las exportaciones de fruta chilena en cada mercado, situación que se analiza en detalle más adelante.

En el Gráfico 2 se presenta la evolución de las exportaciones de las cinco especies de fruta chilena en el marco de ACE No 22 con Bolivia; éste se inicia en el año 1994 con valores de exportaciones cercanos a los 9 millones de dólares, para seguir una marcada tendencia a la baja, llegando a alrededor de 3 millones de dólares en el año 2004. Esto significa que al año 2004 el valor de las exportaciones de fruta chilena a Bolivia se ha reducido a un tercio considerando los valores originales del año 1994.

Para el año 2004, el comercio de fruta con Bolivia es dominado en un $89 \%$ de las ventas por manzana, un $8,9 \%$ por la uva, un $2 \%$ por el kiwi, los volúmenes de palta y pera son muy bajos.

En el Gráfico 3 se presenta la evolución de las exportaciones de las especies chilenas en estudio, dentro del marco de ACE $\mathrm{N}^{\circ} 23$ con Venezuela, que se inicia en el año 1993 con valores de exportaciones cercanos a los 13 millones de dólares, para seguir creciendo hasta alcanzar el máximo histórico de 30 millones de dólares en el año 2001 y de ahí continuar con una fuerte caída donde se alcanza el mínimo en el año 2003 con 5 millones de dólares, para recuperarse levemente en el año 2004 a 9 millones de dólares.

Para el año 2004, el comercio de fruta con Venezuela es dominado en un $70 \%$ por las ventas de manzana, un $23,7 \%$ por pera, un $5,1 \%$ por uva, un $1 \%$ de kiwi y no se registran ventas de palta.

En el Gráfico 4 se presenta la evolución de las exportaciones de las cinco especies de fruta chilena en el marco de ACE N ${ }^{\circ} 24$ con Colombia, éste se inicia en el año 1994 con valores de exportaciones cercanos a los 19 millones de dólares, para seguir creciendo hasta alcanzar el máximo histórico de 38 millones de dólares en el año 1997 y de ahí continuar con una caída y mantenerse en el rango entre los 26 a 30 millones de dólares, mostrando una mayor estabilidad en torno a esta cifra y destacar a Colombia como un importante destino de las exportaciones frutícolas chilenas.

En el año 2004, el comercio de fruta con Colombia es dominado en un $70 \%$ por las ventas de manzana, un $15,3 \%$ de pera, un $13,1 \%$ de uva, un $1,9 \%$ de kiwi y no se registran ventas de palta.

En el Gráfico 5 se presenta la evolución de las exportaciones de las especies en estudio de fruta chilena en el marco de ACE $\mathrm{N}^{\circ} 32$ con Ecuador, éste se inicia en el año 1995 con valores de exportaciones cercano a los 10 millones de dólares, para seguir creciendo hasta alcanzar el máximo histórico de 24 millones de dólares en el año 1998 y de ahí continuar con una caída hasta 9 millones de dólares y comenzar a crecer y recuperarse en el año 2004 llegando las exportaciones a 25 millones de dólares.

En el año 2004, el comercio de fruta con Ecuador es dominado en un $67,4 \%$ por las ventas de manzana, un $22,8 \%$ de uva, un $8 \%$ de pera, un 


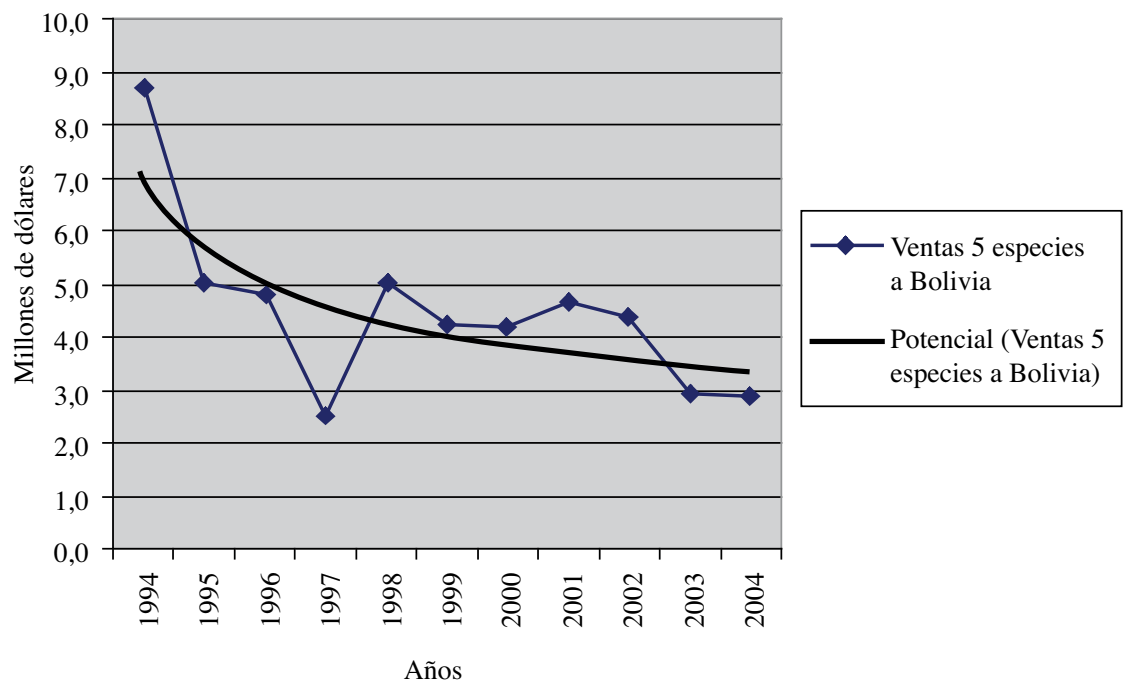

Gráfico 2. Evolución de las ventas de las cinco principales especies frutales chilenas en el marco del ACE 22 con Bolivia entre los años 1994-2004.

(Expresado en millones de dólares)

Fuente: Elaboración propia sobre cifras de ODEPA y Aduanas de Chile, 1994-2004

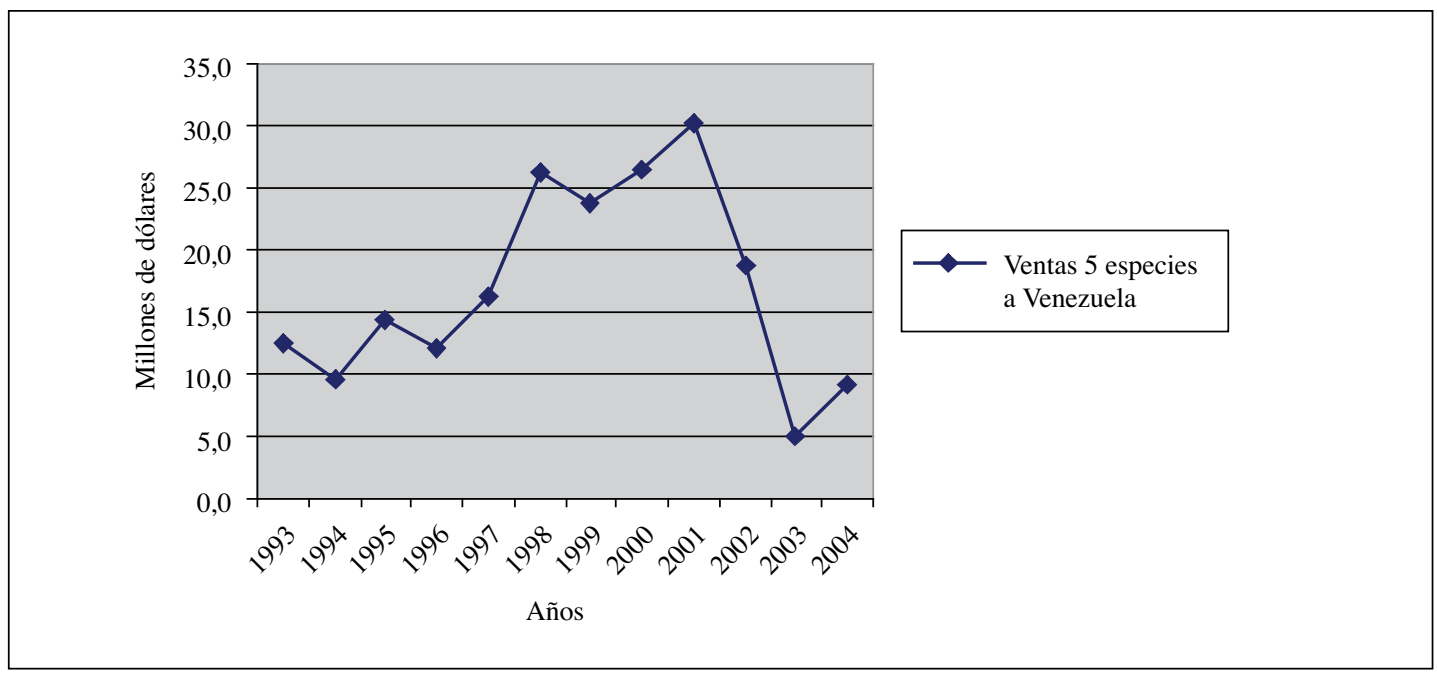

Gráfico 3. evolución de las ventas de las cinco principales especies frutales chilenas en el marco del ACE 23 con Venezuela entre los años 1993-2004.

(Expresado en millones de dólares)

Fuente: Elaboración propia sobre cifras de ODEPA y Aduanas de Chile, 1994-2004.

$1,7 \%$ de kiwi y se registran escasas exportaciones de palta.

En el Gráfico 6 se presenta la evolución de las exportaciones de las cinco especies de fruta chilena en el marco de $\mathrm{ACE} \mathrm{N}^{\circ} 35$ con el grupo de países del MERCOSUR. Las ventas en relación a este acuerdo se iniciaron con gran expectativa en el año 1996 con valores de exportaciones cercano a los 72 millones de dólares, para seguir un comportamiento decreciente llegando las exportaciones de 


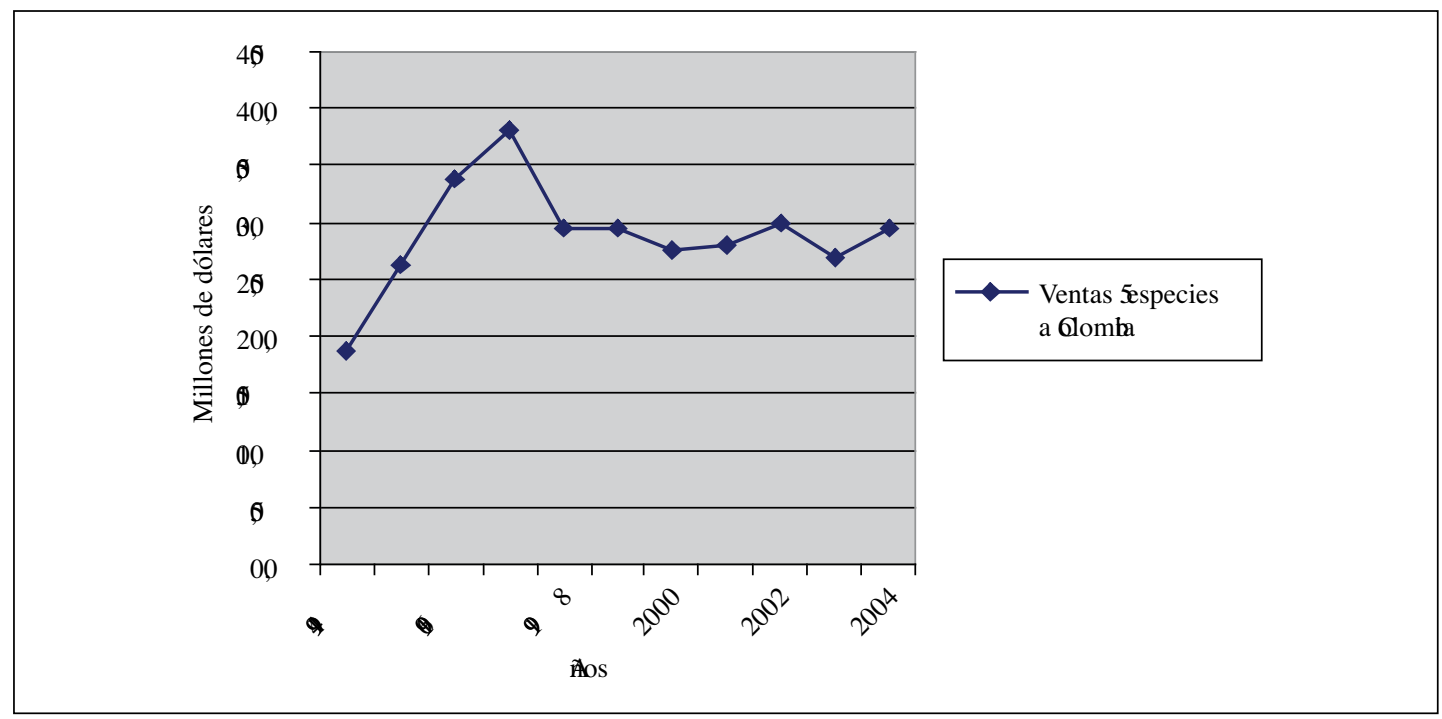

Gráfico 4. Evolución de las ventas de las cinco principales especies frutales chilenas en el marco del ACE 24 con Colombia entre los años 1994-2004.

(Expresado en miles de dólares)

Fuente: Elaboración propia sobre cifras de ODEPA y Aduanas de Chile, 1994-2004.

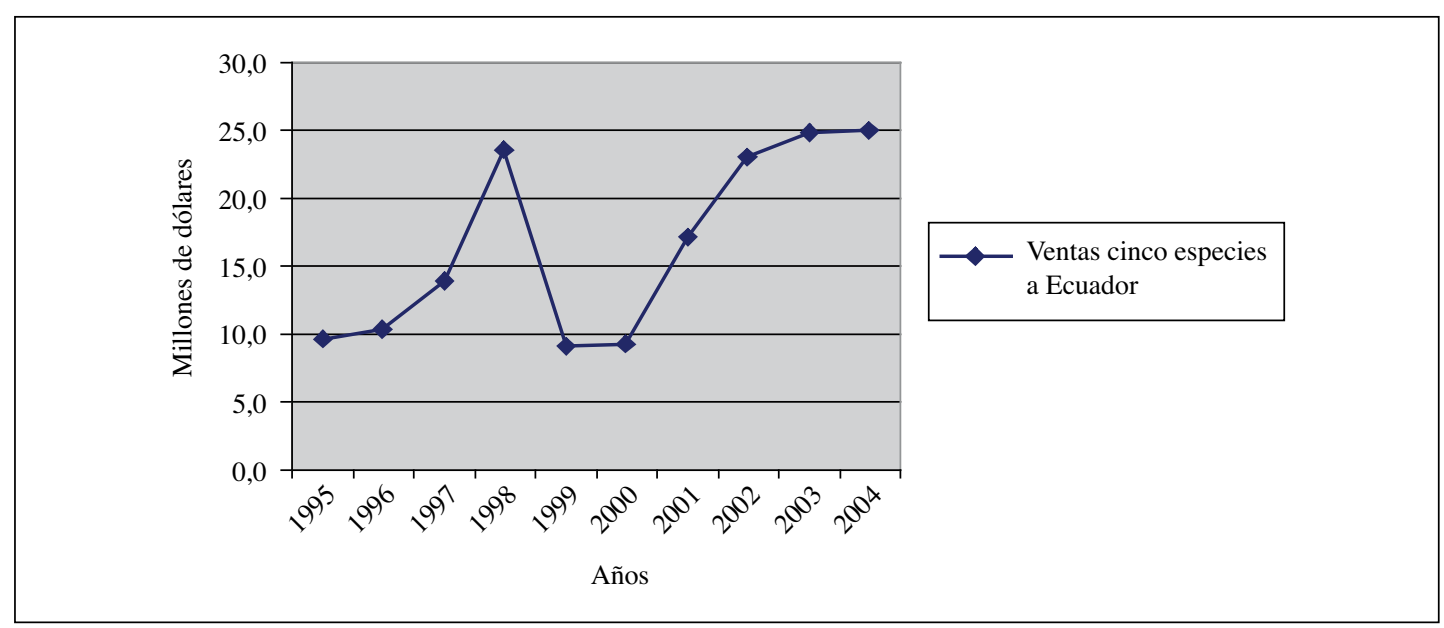

Gráfico 5. Evolución de las ventas de las cinco principales especies frutales chilenas en el marco del ACE 32 con Ecuador entre los años 1995-2004

(Expresado en miles de dólares)

Fuente: Elaboración propia sobre cifras de ODEPA y Aduanas de Chile, 1995-2004.

fruta chilena al bloque del MERCOSUR a valores cercanos a los 12 millones en el año 2004. En este acuerdo es fundamental el comportamiento que registran tanto Brasil con el $70 \%$ de las compras y Argentina con el 27\%, como socios principales del MERCOSUR, mientras que el comportamiento de
Uruguay $(1,77 \%)$ y Paraguay $(1,43 \%)$ es de carácter secundario para las compras de fruta chilena para la serie estudiada entre los años 1996-2004, (Tapia 2006).

En el año 2004, el comercio de fruta con el MERCOSUR es dominado en un $37,6 \%$ por las 


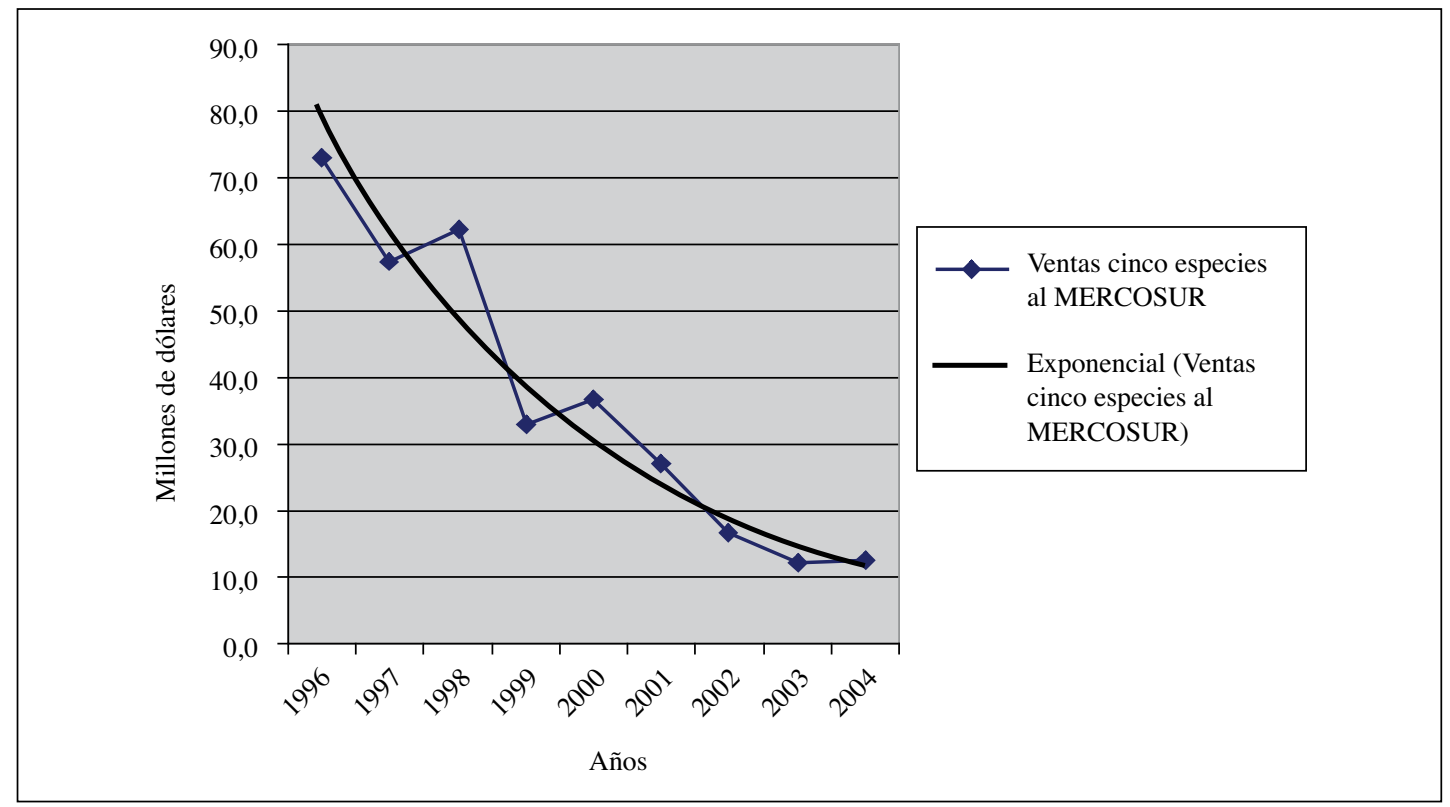

Gráfico 6. Evolución de las ventas de las cinco principales especies frutales chilenas en el marco del ACE 35 con MERCOSUR entre los años 1996-2004.

(Expresado en miles de dólares)

Fuente: Elaboración propia sobre cifras de ODEPA y Aduanas de Chile, 1996-2004

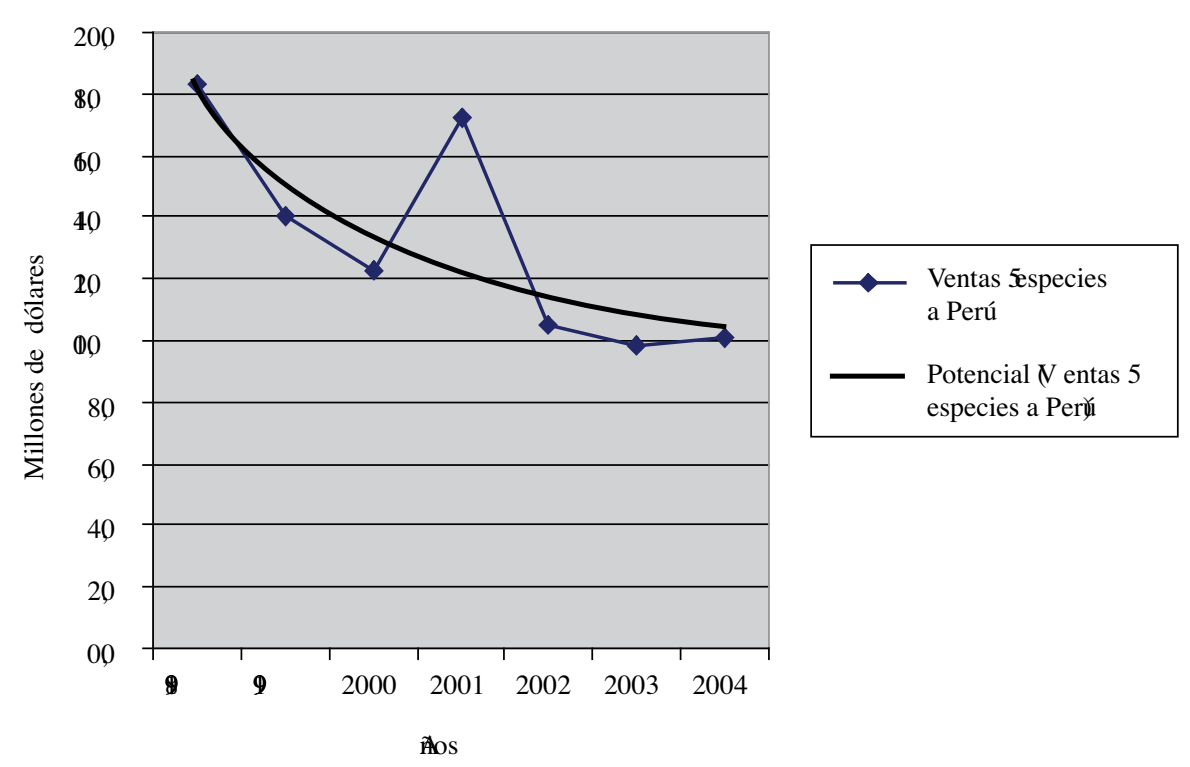

Gráfico 7. Evolución de las ventas de las cinco principales especies frutales chilenas en el marco del ACE 38 con Perú entre los años 1997-2004.

(Expresado en miles de dólares)

Fuente: Elaboración propia sobre cifras de ODEPA y Aduanas de Chile, 1998-2004. 
ventas de kiwi, un $25,15 \%$ por las ventas por manzana, un $18,1 \%$ de uva, un $12,9 \%$ de pera, $y$ un $6 \%$ de palta. Es importante destacar las ventas de peras chilenas en el MERCOSUR, con la alta competencia de Argentina, el primer productor de peras del hemisferio sur.

En el Gráfico 7 se presenta la evolución de las exportaciones de las especies de fruta chilena en estudio dentro del marco del $\mathrm{ACE} \mathrm{N}^{\circ} 38$ con Perú; éste se inicia en el año 1997 con valores de exportaciones cercanos a los 18 millones de dólares, para seguir una marcada tendencia a la baja, llegando a alrededor de 12 millones de dólares en el año 2000 y recuperarse hasta 17 millones de dólares en el año 2001, para luego continuar cayendo hasta 10 millones de dólares, valor mínimo de la serie. Esto significa que al año 2004 el valor de las exportaciones de fruta chilena a Perú se ha reducido en un $45 \%$ con relación a los valores originales del año 1998.

En el año 2004, el comercio de fruta con Perú es dominado en un 79,3\% por las ventas de manzana, un $17,9 \%$ de pera, un $1,6 \%$ de uva, un $1,6 \%$ de kiwi y no se registran ventas de palta.

En el Gráfico 8 se presenta la evolución de las ventas para las especies frutales analizadas para el conjunto de los distintos Acuerdos de
Complementación Económica con los países de América del Sur, para el año 2004. El ACE con Colombia es el acuerdo que domina las ventas con cerca de 30 millones de dólares, seguido del ACE con Ecuador con 25 millones de dólares, los ACE con Perú, Venezuela y MERCOSUR se encuentran en torno a los 10 millones de dólares de ventas y finalmente el ACE con Bolivia con un volumen de ventas cercano a los 3 millones de dólares. Este gráfico permite apreciar la presencia de fruta chilena en América del Sur en el año 2004, con diferentes niveles de ventas, donde el MERCOSUR es superado por las ventas a Ecuador y Colombia.

En el Gráfico 9 se presenta la composición de las ventas para el año 2004 para las especies frutales analizadas para el conjunto de los distintos Acuerdos de Complementación Económica con los países de América del Sur. La especie más importante corresponde a la manzana, la cual se ubica con colocaciones próximas a los 58 millones de dólares, le siguen la uva y pera con similares valores en torno a los 12 millones de dólares, luego el kiwi con 4 millones de dólares, cuyas ventas principalmente se concentran en los países del MERCOSUR, la palta en los países de América del Sur presenta colocaciones muy bajas.

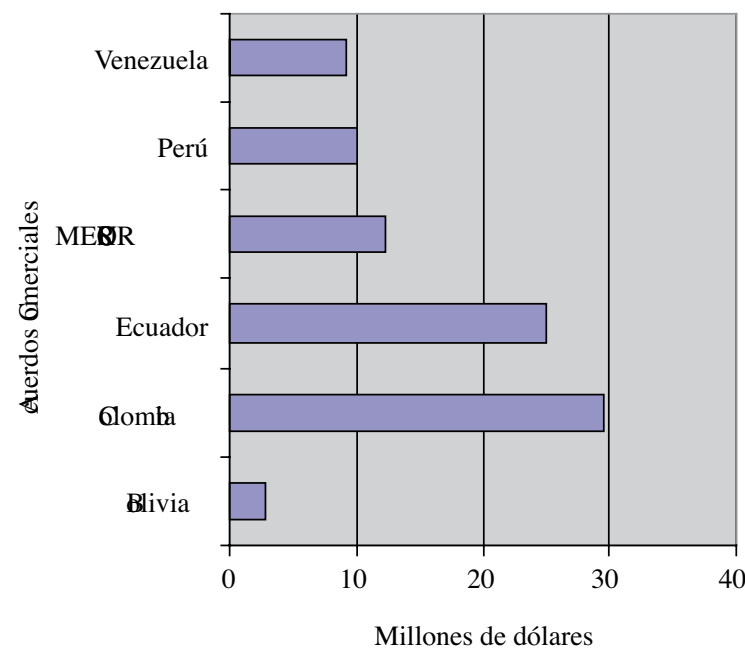

Ventas cinco principales especies futales año 2004 


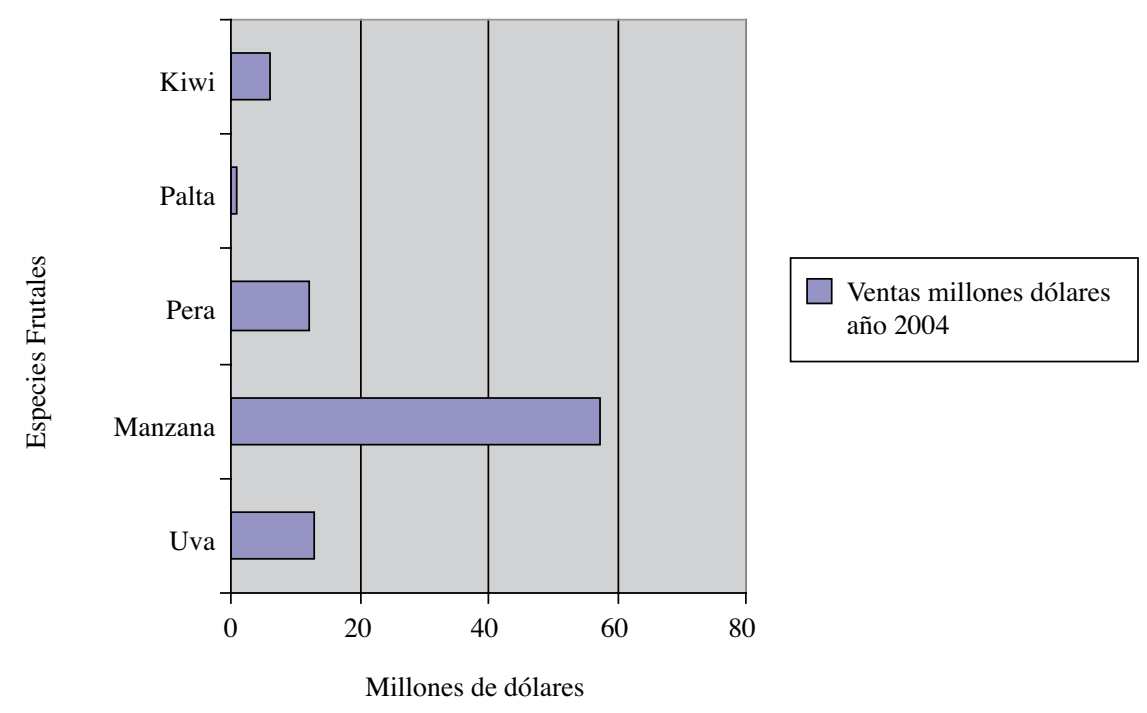

Gráfico 9. Composición de las ventas de las cinco principales especies frutales chilenas en el marco de los ACE con los países de América del Sur en el año 2004.

(Expresado en millones de dólares)

Fuente: Elaboración propia sobre cifras de ODEPA y Aduanas de Chile, 2004.

\section{CONCLUSIONES}

La red de Acuerdos de Complementación Económica que Chile logró articular con los distintos países de América del Sur y el bloque del MERCOSUR ha contribuido de manera efectiva al desarrollo de la fruticultura de exportación, acuerdos que han permitido al país ganar experiencia y colocar importantes volúmenes de fruta en la región.

El análisis particular de los distintos acuerdos muestra diferentes conductas con altos y bajos en las colocaciones, algunas de las cuales se pueden explicar por la variabilidad de las economías de América del Sur. Sin embargo, la fruta chilena ha venido perdiendo presencia en países que presentan importantes niveles de ingresos como Venezuela y Brasil.

En relación al acuerdo con el MERCOSUR, Brasil constituye el principal integrante del pacto y también representa la mayor demanda por fruta chilena.

La situación que se ha alcanzado en relación a los mercados de América del Sur y el MERCOSUR es de alerta para Chile, y requiere prestar atención a la evolución de estos mercados y evitar que otros países productores de la región comiencen a ocupar estos nichos de mercado. A esto se agrega la posibilidad de que terceros países de fuera de la región, como Nueva Zelandia (principal productor de kiwi del hemisferio sur), utilicen a Chile como plataforma de despegue de sus exportaciones de kiwi a América del Sur, al hacer uso del acuerdo P4, donde además Argentina, como principal productor de peras del hemisferio sur, trata de expandir sus ventas en la región.

En esta serie estudiada de exportaciones de fruta chilena a los países de América de Sur, que se inicia en el año 1993 con los ACE, los mejores resultados se han alcanzado con Colombia y Ecuador, países que dominan las exportaciones de fruta desde Chile.

Los nuevos equilibrios que se esperaban del conjunto de acuerdos comerciales con los países de América del Sur han tendido a retrasarse y diluirse en otros acuerdos comerciales que algunos países de la región están implementando o se espera se logren implementar como el ALCA.

Esta experiencia impulsada por Chile, en cuanto a establecer esta red de acuerdos con los países de América del Sur, se está viendo influenciada a partir del año 2003 con la firma de los TLC, con la Unión Europea y el año 2004 con Estados Unidos, que son otros de los más importantes destinos de la fruta chilena. 


\section{LITERATURA CITADA}

CEPAL, 2002. The Chilean strategy of trade liberalization and market access. Santiago, Chile. 49 p.

DIRECON, 1993-2004. Ministerio de Relaciones Exteriores, Acuerdos Complementación Económica firmados por Chile entre 1993-2004, Santiago, Chile. Disponible en: http// www.direcon.cl

HERNÁNDEZ, R.; FERNÁNDEZ, C.; BAPTISTA, P. 1998. Metodología de la Investigación, Editorial Mc Graw-Hill, México.

OFICINA DE ESTUDIOS Y POLÍTICAS AGRARIAS, ODEPA 1993-2004. Base de Datos de exportaciones agropecuarias de Chile. Disponible en: http// www.odepa.cl

DOMÍNGUEZ, J. I. 2000. Una visión prospectiva para la Agricultura Chilena. pp. 91-128. In: La agricultura chilena del 2010, Tres visiones sociopolíticas. ODEPA, Santiago, Chile, 238 p.
MINISTERIO DE AGRICULTURA, MINAGRI, 2000. Una Política de Estado para la Agricultura Chilena, Periodo 2000-2010. Santiago, Chile, 140 p.

MUCHNIK, E.; ERRÁZURIZ F.; DOMÍNGUEZ, J. I. 1996. Efectos de la asociación de Chile al MERCOSUR en el sector agrícola y agroindustrial. Estudios Públicos, 63 (invierno 1996) pp. 113-164.

SEPÚLVEDA, C.; RIVERA, L. 2004. Análisis del impacto económico en la región de O’Higgins de los nuevos acuerdo comerciales de Chile. Ministerio de Planificación, Departamento de Competitividad Regional. 156 p.

TAPIA, I. L. 2006. Evaluación de las condiciones de competitividad del sector frutícola chileno con relación a su inserción al mercado internacional entre los años 1993-2004. Ediciones Universidad de Tarapacá (en prensa). 